\title{
EQUIPE EDITORIAL E AVALIADORES AD HOC
}

\section{EDITORA-CHEFE}

Dra. Greice da Silva Lorenzzetti Andreis

Instituto Federal de Educação, Ciência e Tecnologia do Rio Grande do Sul (IFRS), Campus Caxias do Sul, RS, Brasil

\section{EDITORA ADJUNTA}

Dra. Katia Arcaro

Instituto Federal de Educação, Ciência e Tecnologia do Rio Grande do Sul (IFRS), Campus Caxias do Sul, RS, Brasil

\section{COMISSÃO EDITORIAL}

Ma. Daiane Scopel Boff

Instituto Federal de Educação, Ciência e Tecnologia do Rio Grande do Sul (IFRS), Campus Caxias do Sul, RS, Brasil

\section{Me. Henri Luiz Fuchs}

Instituto Federal de Educação, Ciência e Tecnologia do Rio Grande do Sul (IFRS), Campus Bento Gonçalves, RS, Brasil

Dra. Katia Arcaro

Instituto Federal de Educação, Ciência e Tecnologia do Rio Grande do Sul (IFRS), Campus Caxias do Sul, RS, Brasil

Me. Michelsch João da Silva

Instituto Federal de Educação, Ciência e Tecnologia de Santa Catarina (IFSC), Campus Florianópolis, SC, Brasil

\section{Dr. Nicolau Matiel Lunardi Diehl}

Instituto Federal de Educação, Ciência e Tecnologia do Rio Grande do Sul (IFRS), Campus Canoas, RS, Brasil

\section{Dr. Rodrigo Sychocki da Silva}

Universidade Federal do Rio Grande do Sul (UFRGS), Instituto de Matemática e Estatística (IME), Porto Alegre, RS, Brasil 


\title{
CONSELHO EDITORIAL CONSULTIVO
}

\author{
Dra. Adriana Breda \\ Universitat de Barcelona, Barcelona, Espanha \\ Dr. Carloney Alves de Oliveira
}

Universidade Federal de Alagoas (UFAL), Centro de Educação (CEDU), Campus Aristóteles Calazans Simões, Maceió, AL, Brasil

Dra. Carolina Augusta Assumpção Gouveia

Centro de Ensino Superior de Valença (CESVA/FAA), Valença, RJ, Brasil

Dra. Franciele Corti

Universitat de Barcelona (UB), Campus Mundet, Barcelona, Espanha

Dr. Francisco Regis Vieira Alves

Instituto Federal de Educação, Ciência e Tecnologia do Estado do Ceará (IFCE), Fortaleza, CE, Brasil

Dra. Maria Cristina Caminha de Castilhos França

Instituto Federal de Educação, Ciência e Tecnologia do Rio Grande do Sul (IFRS), Campus Porto Alegre, RS, Brasil

\author{
Dr. Moiseis Cecconello \\ Universidade Federal de Mato Grosso (UFMT), Cuiabá, MT, Brasil \\ Dr. Rene Carlos Cardoso Baltazar Júnior \\ Universidade Federal do Rio Grande (FURG), Rio Grande, RS, Brasil \\ Dr. Wagner Marcelo Pommer \\ Universidade Federal de São Paulo (UNIFESP), Campus Diadema, SP, Brasil
}

\section{EDITORES DE TEXTO}

\author{
Dra. Aline Brustulin Cecchin \\ Universidade de Caxias do Sul (UCS), Caxias do Sul, RS, Brasil
}

Ma. Aline Regina Horbach

Instituto Federal de Educação, Ciência e Tecnologia do Rio Grande do Sul (IFRS), Campus Caxias do Sul, RS, Brasil

Dr. Claudionor Ferreira Araujo

Instituto Federal de Educação, Ciência e Tecnologia do Rio Grande do Sul (IFRS), Campus Caxias do Sul, RS, Brasil

Me. Jorgemar Teixeira

Instituto Federal de Educação, Ciência e Tecnologia do Rio Grande do Sul (IFRS), Campus Caxias do Sul, RS, Brasil

Ma. Manuela Damiani Poletti da Silva

Instituto Federal de Educação, Ciência e Tecnologia do Rio Grande do Sul (IFRS), Campus Caxias do Sul, RS, Brasil 


\title{
APOIO TÉCNICO
}

\section{Srta. Camila Gasparin Magnaguagno}

Instituto Federal de Educação, Ciência e Tecnologia do Rio Grande do Sul (IFRS), Bolsista BICTES/IFRS, Campus Caxias do Sul, RS, Brasil

\section{AVALIADORES AD HOC}

\author{
Dra. Adriana Breda
}

Universitat de Barcelona, Barcelona, Espanha

Dr. Augusto Vieira Cardona

Pontifícia Universidade Católica do Rio Grande do Sul (PUC/RS), Porto Alegre, RS, Brasil

\author{
Dra. Carolina Augusta Assumpção Gouveia \\ Centro de Ensino Superior de Valença (CESVA/FAA), Valença, RJ, Brasil
}

Me. Cassiano Scott Puhl

Pontifícia Universidade Católica do Rio Grande do Sul (PUC/RS), Porto Alegre, RS, Brasil

Dr. Cristiano Lima Hackmann

Universidade Federal do Rio Grande do Sul (UFRGS), Porto Alegre, RS, Brasil

Me. Dailson Evangelista Costa

Universidade Federal do Tocantins (UFT), Palmas, TO, Brasil

\section{Dr. Diego Marcon Farias}

Universidade Federal do Rio Grande do Sul (UFRGS), Departamento de Matemática, Instituto de Matemática e Estatística, Porto Alegre, RS, Brasil

\section{Ednei Luís Becher}

Instituto Federal de Educação, Ciência e Tecnologia do Rio Grande do Sul (IFRS), Campus Osório, RS, Brasil

\section{Eimard Gomes Antunes do Nascimento \\ Universidade de Aveiro, Portugal}

Dr. Fabiano Carlos Cidral

Instituto Federal de Educação, Ciência e Tecnologia de Santa Catarina (IFSC), Campus de Florianópolis, SC, Brasil

Dra. Franciele Corti

Universitat de Barcelona (UB), Campus Mundet, Barcelona, Espanha

\section{Dr. Francisco Regis Vieira Alves}

Instituto Federal de Educação, Ciência e Tecnologia do Estado do Ceará (IFCE), Fortaleza, CE, Brasil 


\section{Me. Jeremias Stein Rodriguês}

Instituto Federal de Educação, Ciência e Tecnologia de Santa Catarina (IFSC), Florianópolis, SC, Brasil

$$
\text { Dr. João Cândido Moraes Neves }
$$

Instituto Federal de Educação, Ciência e Tecnologia do Rio Grande do Sul (IFRS), Campus Caxias do Sul, RS, Brasil

$$
\text { Dr. João Francisco Silva Filho }
$$

Universidade da Integração Internacional da Lusofonia Afro-Brasileira (UNILAB), Redenção, CE, Brasil

$$
\text { Dr. José Luiz de Souza Pio }
$$

Universidade Federal do Amazonas (UFAM), Instituto de Computação, Manaus, AM, Brasil

$$
\text { Ma. Juliana Fronza }
$$

Universidade Estadual do Rio Grande do Sul (UERGS), Unidade Bento Gonçalves, RS, Brasil

\section{Me. Manuel Bandeira dos Santos Neto}

Instituto Federal de Educação, Ciência e Tecnologia do Estado do Ceará (IFCE), Fortaleza, CE, Brasil

$$
\text { Dr. Marcos Craizer }
$$

Pontifícia Universidade Católica do Rio de Janeiro (PUC-Rio), Rio de Janeiro, RJ, Brasil

\section{Me. Nícolas Müller}

Instituto Federal de Educação, Ciência e Tecnologia do Rio Grande do Sul (IFRS), Campus Caxias do Sul, RS, Brasil

\section{Me. Nitiele Medeiros Contessa}

Instituto Federal de Educação, Ciência e Tecnologia do Rio Grande do Sul (IFRS), Campus Caxias do Sul, RS, Brasil

$$
\text { Dr. Paulo Mendes de Carvalho }
$$

Universidade Federal de Santa Catarina (UFSC), Santa Catarina, SC

\section{Dr. Rafael Cavalheiro}

Universidade Federal do Rio Grande (FURG), Rio Grande, RS, Brasil

\section{Dr. Rene Carlos Cardoso Baltazar Júnior}

Universidade Federal do Rio Grande (FURG), Rio Grande, RS, Brasil

\section{Dra. Rúbia Emmel}

Instituto Federal de Educação, Ciência e Tecnologia Farroupilha, Campus Santa Rosa, RS, Brasil

$$
\text { Dr. Wagner Marcelo Pommer }
$$

Universidade Federal de São Paulo (UNIFESP), Campus Diadema, SP, Brasil

Dra. Zulma Elizabete de Freitas Madruga

Universidad de Los Lagos, Osorno, Chile 